\title{
GASTO NA FUNÇÃO DESPORTO E LAZER PELAS PREFEITURAS DO ESTADO DO MATO GROSSO DE 2002 A 2011
}

\author{
${ }^{1}$ Edmilson Santos \\ 2 Vilde Gomes Menezes \\ ${ }^{3}$ Maria José Carvalho \\ ${ }^{4}$ Riller Silva Reverdito
}

\section{Resumo}

Objetivo: Analisar o gasto na Função Desporto e Lazer (FDL) das prefeituras do estado do Mato Grosso de 2002 a 2011, contemplando a adesão e o volume do gasto por classe populacional.

Metodologia: Estudo de natureza descritivo-exploratória utilizando estatística descritiva. Os dados referentes à FDL foram capturados diretamente no Sistema de Informações Contábeis e Fiscais do Setor Público Brasileiro e, então, deflacionados.

Relevância: Os municípios têm sido grandes players do financiamento das políticas públicas de esporte e de lazer, mas poucos estudos têm descrito essa realidade.

Resultados e Contribuições: Há forte inclinação das prefeituras do estado do Mato Grosso em realizar gastos na FDL de forma crescente nas políticas de esporte e de lazer. O desafio agora é compreender quais variáveis impactam nesse comportamento.

Palavras-chave: Financiamento. Políticas Públicas. Esporte. Lazer

\section{Como referenciar em APA:}

Santos, E., Menezes, V. G., Carvalho, M. J., \& Reverdito, R. S. (2019). Gasto na função do desporto e lazer pelas prefeituras do Estado do Mato Grosso de 2002 a 2011. PODIUM Sport, Leisure And Tourism Review, 8(2), 253-262. https://doi.org/10.5585/podium.v8i2.11243

\footnotetext{
${ }^{1}$ Universidade Federal do Vale do São Francisco UNIVASF, Pernambuco, (Brasil). E-mail: edmilson.santos@univasf.edu.br Orcid: http://orcid.org/0000-0003-3805-2319

${ }^{2}$ Universidade Federal de Pernambuco - UFPE, Pernambuco, (Brasil). E-mail: vildemenezes@ hotmail.com Orcid: https://orcid.org/0000-0002-4006-2711

${ }^{3}$ Faculdade de Desporto da Universidade do Porto - FADEUP, Porto, (Portugal). E-mail: mjc @ fade.up.pt Orcid: https://orcid.org/0000-0002-4975-0115

${ }^{4}$ Universidade do Estado de Mato Grosso - UNEMAT, Mato Grosso, (Brasil). E-mail: rsreverdito@unemat.br Orcid: https://orcid.org/0000-0003-0556-9151
} 


\title{
EXPENDITURE ON THE FUNCTION OF SPORT AND LEISURE BY THE MATO GROSSO STATE COUNCIL FROM 2002 TO 2011
}

\begin{abstract}
Objective. Analyze the expenditure in the Sports and Leisure Function (SLF) of municipalities from the state of Mato Grosso from 2002 to 2011, considering adherence and volume of spending per population class.

Methodology. Descriptive and exploratory study using descriptive statistics. Data related to the SLF were collected directly in the Brazilian Public Sector Accounting and Tax Information System and then deflated.

Relevance. Municipalities have been major players in the financing of sport and leisure public policies, but few studies have been describing such reality.

Results and Contributions. There is a strong inclination on the part of municipalities from Mato Grosso to spend more and more on sports and leisure policies in the SLF. The challenge now is to understand which variables impact this behavior.
\end{abstract}

Keywords: Funding. Public Policies. Sport. Leisure.

GASTOS EN LA FUNCIÓN DE DEPORTE Y OCIO DEL CONSEJO DE ESTADO DE MATO GROSSO DE 2002 A 2011

\section{Resumen}

Objetivo. Analizar el gasto en la Función de Deportes y Ocio (SLF) de los municipios del estado de Mato Grosso de 2002 a 2011, considerando la adherencia y el volumen de gasto por clase de población.

Metodología. Estudio descriptivo y exploratorio utilizando estadística descriptiva. Los datos relacionados con el SLF se recopilaron directamente en el Sistema de Información Fiscal y Contable del Sector Público brasileño y luego se desinflaron.

Relevancia. Los municipios han sido actores importantes en la financiación de políticas públicas de deporte y ocio, pero pocos estudios han descrito esta realidad.

Resultados y Contribuciones. Existe una fuerte inclinación por parte de los municipios de Mato Grosso a gastar cada vez más en políticas deportivas y de ocio en el SLF. El desafío ahora es comprender qué variables impactan este comportamiento.

Palabras clave: Financiamiento. Políticas públicas. Deporte. Ocio. 


\section{Introdução}

O tema do gasto público na área das políticas públicas de esporte e de lazer tem ganhado espaço dentro das policy communities da área de políticas públicas de esporte e de lazer, de acordo com Santos e Santos (2018). De alguma forma essa produção está associada ao peso político que a agenda ganhou com a criação do Ministério do Esporte. A existência de uma instituição federal responsável por pensar as políticas de esporte da federação permitiu aguçar os olhares, principalmente acadêmicos, aos governos, de forma a compreender o desempenho na formulação, implementação e avaliação das políticas de esporte e de lazer.

A agenda do governo federal passou a ser alvo de escrutínio acadêmico e diversos trabalhos na área passaram a ser estudados. Parte significativa dos trabalhos estão preocupados em avaliar o desempenho do ator central do nosso federalismo, o governo federal. ${ }^{5}$ Nessa perspectiva, se destacam as análises de Veronez (2005), Castro (2016), Almeida e Marchi Jr. (2010), Athayde (2014), Athayde, Mascarenhas e Salvador (2015), Castro, Scarpin e Souza (2017) e Corrêa et al. (2014), por exemplo. As análises dos entes subnacionais também têm recebido atenção. Furtado (2016) analisou o estado de Santa Catarina e Silva e Santana e Silva (2015), o estado da Bahia. Recentemente tem surgido uma forte preocupação em analisar o desempenho das prefeituras, como nos trabalhos de Ribeiro (2012), Silva, Couto e Santos (2014), Venturim, Borges e Silva (2013), Santos, Canan e Starepravo (2018) e Grasso e Isayama (2017).

No nível internacional a preocupação com o gasto na área das políticas de esporte também tem chamado atenção dos analistas. Hoekman, Van Der Roest e Van Der Poel (2018) analisaram os gastos dos municípios holandeses. A forma como ele se dá na esfera local tem sido preocupação de diferentes estudiosos: Pavlik (2013) e Pavlik e Vries (2014) na República Tcheca; Widdop (2018) na Inglaterra; Yuval (2011) em Israel; e Taks e Kêsenne (2000) na Bélgica. As preocupações com o gasto do esporte de elite também sido debatidas (Green, 2007; Ronan, 2015).

Considerando a especificidade da gestão do Estado brasileiro, de forma inaugural alguns trabalhos procuram compreender as relações federalistas. As relações verticais são tratadas na obra de Diniz e Silva
(2016) sobre o ICMS esportivo. Santos, Starepravo e Souza Neto (2015) analisaram a descentralização do Programa Segundo Tempo na região Nordeste. O trabalho de Santos e Hirata (2017) analisa o desempenho das prefeituras quanto ao gasto das políticas públicas de esporte e de lazer por níveis de Índice de Desenvolvimento Humano (IDH).

Dadas as características de nosso federalismo (com 3 entes federados), temos diversas possibilidades de cenários à implementação das políticas de esporte e de lazer. Em um deles, governo federal, 27 Unidades da Federação e 5.570 municípios, totalizando 5.598 atores governamentais, têm a possibilidade de produzirem suas próprias agendas políticas. Noutro, acontecem as relações intergovernamentais. O governo federal pode se relacionar com os entes subnacionais (estados e municípios) e os governos estaduais, com os municípios. Portanto, o arranjo federativo possibilita atuação isolada dos entes federados ou em cooperação com outros.

No entanto, pouco se explora o viés (incentivo) que possibilita que os entes federados nada façam em termos de políticas públicas de esporte e de lazer. A despeito do artigo 217 da Constituição Federal de 1988 (CF/88), o Estado brasileiro não ofereceu instrumentos normativos que obriguem os diferentes governos a implementarem essas políticas ${ }^{6}$. Esse tema ficou completamente ausente das atribuições privativas ou exclusivas da União e dos municípios (artigos 21 e 30 da CF/88, respectivamente), como também das competências comuns (artigo 23 da $\mathrm{CF} / 88$ ). Por outro lado, por força da constituição, nenhuma instância de governo (poder executivo dos 3 entes federados) está obrigada a formular e implementar políticas públicas de esporte e de lazer.

Afonso e Lobo (1996) e Melo (1998) já destacavam que a ambiguidade de competências do federalismo brasileiros em diferentes áreas tem sido um dos problemas enfrentados para melhor coordenação da oferta de políticas públicas, principalmente as sociais. No entanto, no caso do esporte, não há previsão dentro da constituição que indique de quem é a responsabilidade (poder executivo) da aplicação do direito. Esse problema foi parcialmente contornado quando os congressistas indicaram como ator principal e estratégico à aplicação d direito o poder legislativo (inciso 9 do artigo $24 \mathrm{da} \mathrm{CF} / 88$ ).

\footnotetext{
5 Nesse item, consideramos tal como Rocha (2011), a

${ }^{6}$ Para uma visão mais ampla, sugere-se a obra Starepravo dimensão conflituosa acerca do conceito de federalismo.
} 
Portanto, quando se trata de implementação da política, o arcabouço institucional do federalismo brasileiro tem forte viés à inação ${ }^{7}$ das políticas de esporte e de lazer. No entanto, a atuação do Ministério do Esporte na formulação de políticas permite pensar que o mesmo protagonismo também pode estar acontecendo no nível municipal. Em áreas em que há constrangimentos no âmbito da federação impondo certos comportamentos aos atores, como na educação e na saúde, não há possibilidade de inação. Mas em áreas não reguladas esse é um comportamento possível de ser alcançado.

Desde 1999, o Ministério da Gestão e do Orçamento publicou portaria (Portaria $\mathrm{n}^{\circ} 42$ ) criando taxonomia do gasto orçamentário dos governos. Dentre as unidades de gasto é possível observar que uma foi proposta para dimensionar o gasto em políticas públicas de esporte e de lazer: a Função Desporto e Lazer (FDL). A FDL é subdividida em 5 subfunções: desporto comunitário, desporto rendimento, lazer, demais funções do desporto e, a partir de 2016, administração geral.

Há dois modelos que explicam o comportamento dos atores na oferta de políticas em estados federados. A descentralização top-down e bottom up. Para Sabatier (1986) a descentralização top-down permite compreender como políticas formuladas por um ente superior são implementadas pelos governos locais, no caso aqui o município. Os programas Segundo Tempo e Esporte e Lazer na Cidade são dois exemplos de políticas sociais na área do esporte e do lazer que são descentralizadas aos municípios. A descentralização bottom up permite compreender a implementação da política formulada pelo ente $\mathrm{local}^{8}$. O município pode ser uma correia de transmissão de uma política de outro nível de governo ou implementador de sua própria agenda.

A literatura federalista indica que os municípios possuem diferentes capacidades de implementação de suas políticas. No entanto, como revela Santos (2011), apenas as cidades acima de $100 \mathrm{mil} / \mathrm{hab}$. têm condições de experimentarem a autonomia alocativa (formulação da política bottom up). Já os municípios

\footnotetext{
${ }^{7}$ Compõe uma das opções do gestor público, deixar de fazer algo sobre um determinado tema (Souza, 2003). É a escolha de nada fazer que é considerada aqui como inação. ${ }^{8}$ Alguns estudos têm sido produzidos no Brasil no sentido de compreender os resultados da descentralização topdown junto as prefeituras, como Santos, Starepravo e Hirata (2018), Santos (2017) e Santos (2016).

${ }^{9}$ Consiste em uma ação programática do Ministério do Esporte voltada para o fomento à pesquisa estruturada
}

pequenos, especialmente aqueles até $50 \mathrm{mil} / \mathrm{hab}$, por terem baixa dinâmica econômica são dependentes do governo federal (Speroto, 2009; Moraes, 2006). Sendo assim, tem menor capacidade de experimentar a autonomia alocativa.

Há uma tripla complexidade para se compreender o gasto público nas políticas de esporte no Brasil pelo fato de existirem 3 entes federados. Apesar de terem surgido estudos que abordam a análise local, estudos focais, carecemos de análises comparativas no âmbito estadual, regional e nacional. Alguns estados já foram contemplados nessa perspectiva como os estados da Bahia (Santos, Starepravo, \& Canan, 2018), Espírito Santo (Santos \& Santos, 2018), Piauí (Santos \& Starepravo, 2018), Maranhão (Santos, 2018).

Considerando a estrutura federativa brasileira, precisamos avançar no sentido de compreender de forma comparativa o gasto público na área do esporte e do lazer em outras unidades da federação. A escolha do estado do Mato Grosso se deve pela parceria realizada entre 2 Centros de Pesquisa em Políticas Públicas de Esporte e de Lazer da Rede Cedes ${ }^{9}$. O centro do Piauí, que estuda a descentralização de políticas de esporte, e o do Mato Grosso, que busca aprimorar o nível de informação sobre as políticas públicas de esporte e de lazer.

Nesse sentido, o presente trabalho buscou conhecer o gasto descentralizado (bottom up) nas políticas públicas de esporte e de lazer no estado do Mato Grosso de 2002 a $2011^{10}$. O intervalo de tempo se refere aos 10 primeiros anos em que os municípios passaram a registrar o gasto nas políticas de esporte e de lazer através da FDL. A pergunta de pesquisa pode ser compreendida nos seguintes termos: (a) Qual foi a adesão e o gasto total e por classe populacional na Função Desporto e Lazer (FDL) por parte das prefeituras de Mato Grosso?

\section{Metodologia}

O presente estudo de natureza descritivoexploratória buscou fazer um balanço do gasto da FDL no estado do Mato Grosso por parte das

enquanto uma rede de Centros de Desenvolvimento de Esporte Recreativo e de Lazer (REDE CEDES). Atualmente, são 27 Centros, alcançando todos os Estados brasileiros, mais o Distrito Federal

${ }^{10}$ É mister destacar, que outras unidades da federação tem sido pautas por uma agenda de estudo referente a temática em tela, que estamos realizando desde 2015. 
prefeituras de 2002 a 2011. Em 2002, a Secretaria do Tesouro Nacional começa a disponibilizar no Sistema de Informações Contábeis e Fiscais do Setor Público Brasileiro (SICONFI) os gastos realizados pelas prefeituras na FDL. Dada a necessidade de compreender o comportamento consolidado das prefeituras ao longo do tempo, escolhemos analisar os 10 primeiros em investimentos liquidados nas políticas de esporte e de lazer.

Os recursos investidos na FDL foram capturados junto ao site do Tesouro Nacional (https://siconfi.tesouro.gov.br/siconfi/pages/publi), nos dados referentes às contas anuais, no Sistema de Informações Contábeis e Fiscais do Setor Público Brasileiro (SICONFI), entre os dias 3 e 8 de março de 2018. A listagem dos 141 municípios do estado do Mato Grosso foi extraída diretamente do site do Instituto Brasileiro de Geografia e Estatística (IBGE). O conjunto dos dados compôs planilha Excel com o nome das cidades e os valores referentes às despesas liquidadas na FDL de 2002 a 2011. A utilização da estatística descritiva permitiu identificar os valores totais e percentuais envolvidos.

Para garantir a comparabilidade das informações fiscais, os valores referentes à FDL foram deflacionados pelo Índice de Preços ao Consumidor Ampliado (IPCA) em dois recortes temporais. No primeiro, os valores foram deflacionados para melhor compreender os gastos na FDL de 2002 a 2011. Para ajustarmos a classificação por classe populacional (CP) estabelecemos os seguintes critérios (a partir da projeção populacional inserida no SICONFI pelos municípios para o ano de 2002):

CP1: cidades $<50 \mathrm{mil} / \mathrm{hab}$.; $(95,7 \% ; \mathrm{n}=135)$

$\mathrm{CP} 2$ : cidades $\geq$ de $50 \mathrm{mil} / \mathrm{hab}$. e $<100 \mathrm{mil} / \mathrm{hab}$. $(2,1 \% ; \mathrm{n}=3)$

CP3: cidades $\geq$ de $100 \mathrm{mil} / \mathrm{hab} .(2,1 \% ; \mathrm{n}=3)$

A adesão foi aferida por 4 níveis: I - inação, quando os municípios não realização gasto na FDL; BA - baixa adesão, quando a adesão se dá abaixo de $50 \%$; MA, quando a adesão se dá superior e $50 \%$ e menos que 80\%; AA - alta adesão, quando os municípios realizam adesão superior a $80 \%$.

\section{Análise dos Resultados}

Ao observarmos a adesão das prefeituras do estado do Mato Grosso ao gasto na FDL (Tabela 1) constatamos que: (a) os níveis de adesão ao gasto se mantiveram superiores a $80 \%$ (AA) em $70 \%$ do período; (b) é possível perceber uma nítida mudança de comportamento das prefeituras na segunda metade da década. Enquanto na primeira metade a média de adesão ficou em $80,7 \%$, impactado fortemente pela adesão de 2006, na segunda esse percentual foi de $91,77 \%$, demonstrando a elevação da adesão na segunda metade do período; (c) considerando o estoque do período, ano de entrada e ano de saída, houve importante redução da inação. Ela partiu de 25,5\% em 2002 para 7,1\% em 2011. Enquanto a média de I ficou em $22 \%$ nos 4 primeiros anos da série, nos quatro últimos ficou em 7,3\%. Ou seja, houve diminuição da inação ao longo do período, apesar da variação entre 2006 para 2007 e 2010 para 2011; (d) o número de prefeituras com $\mathrm{AA}$ foi de $78,72 \%(n=111)$, MA $17 \%(n=24)$ e BA 4,3 $(n=6)$; (e) e apenas uma cidade, Rondolândia, com 3538 no Censo de 2010 do Instituto Brasileiro de Geografia e Estatística (IBGE).

Portanto, apesar dos incentivos do federalismo brasileiro em direção à inação, as prefeituras do estado do Mato Grosso preferem investir recursos na implementação de políticas públicas de esporte e de lazer. Se considerarmos que para 49,6\% ( $\mathrm{n}=70)$ realizaram gastos em todo o período e o nível de AA, é possível afirmar que as prefeituras do estado participam intensamente do gasto na FDL. Há elementos institucionais (instituições aqui deve ser entendido no sentido que é dado pelo neoinstitucionalismo ${ }^{11}$ ) que pressionam os policy makers (decisores) a terem postura ativa em relação a essa agenda.

\footnotetext{
${ }^{11}$ Corrente de análise das políticas públicas que destaca o papel das normas, das ideias e da cultura na formatação das
}

políticas. Para análise mais detalhada da corrente, ver Hall e Tailor (2003). 
Tabela 1. Níveis de adesão ao gasto na FDL por parte das prefeituras do estado do Mato Grosso de 2002 a 2011.

\begin{tabular}{|c|c|c|c|c|c|c|c|c|c|c|}
\hline Adesão & 2002 & 2003 & 2004 & 2005 & 2006 & 2007 & 2008 & 2009 & 2010 & 2011 \\
\hline $\mathrm{N}$ & 105 & 110 & 112 & 113 & 129 & 124 & 128 & 132 & 132 & 131 \\
\hline$\%$ & 74,5 & 78,0 & 79,4 & 80,1 & 91,5 & 87,9 & 90,8 & 93,6 & 93,6 & 92,9 \\
\hline Adesão & MA & MA & MA & AA & AA & AA & AA & AA & AA & AA \\
\hline
\end{tabular}

Fontes. Dados da pesquisa.

A literatura federalista aponta que a liberdade alocativa está diretamente relacionado com a capacidade dos municípios terem condições fiscais para fazer frente aos gastos. Como sabemos, os gastos na FDL não são estruturados por norma superior, como são na educação e na saúde. As cidades da CP1 são as que possuem maiores limitações ficais para garantirem autonomia alocativa, como revela Speroto (2009) e Moraes (2006). Aqueles com mais de 100 mil/hab., possuem melhores condições fiscais para experimentarem a autonomia. No entanto, como podemos observar na tabela 2, os municípios da CP1 e CP3 se igualam e tiveram o mesmo desempenho quando se trata de distribuição por níveis de adesão. Os 2 grupos obtiveram $60 \%$ de adesão em AA e $40 \%$ em MA. Portanto, para esse nível de análise não houve diferença entre os 2 grupos.

As cidades do nível intermediário, do ponto de vista da classe populacional (CP2), apresentaram melhores resultados do que $\mathrm{CP} 1$ e $\mathrm{CP} 3$. Na $\mathrm{CP} 2$ a adesão no nível AA foi de $80 \%$ e MA, 20\%. Portanto, há variáveis importantes que atuam na $\mathrm{CP} 2$ que permitiu obterem melhores resultados em termo de adesão. Apesar dessas diferenças, em mais de 50\% dos cenários de todas as CPs houve AA ao gato na FDL.

Tabela 2. Níveis de adesão ao gasto na FDL por classe populacional (CP).

\begin{tabular}{|c|c|c|c|c|c|c|c|c|c|c|c|}
\hline $\mathrm{CP}$ & Adesão & 2002 & 2003 & 2004 & 2005 & 2006 & 2007 & 2008 & 2009 & 2010 & 2011 \\
\hline \multirow{3}{*}{ CP1 } & $\mathrm{N}$ & 100 & 104 & 106 & 107 & 124 & 119 & 123 & 127 & 127 & 125 \\
\hline & $\%$ & 74,1 & 77,0 & 78,5 & 79,3 & 91,9 & 88,1 & 91,1 & 94,1 & 94,1 & 92,6 \\
\hline & Adesão & MA & MA & MA & MA & $\mathrm{AA}$ & AA & AA & AA & $\mathrm{AA}$ & AA \\
\hline \multirow{3}{*}{$\mathrm{CP} 2$} & $\mathrm{~N}$ & 3 & 3 & 3 & 3 & 3 & 3 & 3 & 2 & 2 & 3 \\
\hline & $\%$ & 100 & 100 & 100 & 100 & 100 & 100 & 100 & 66,7 & 66,7 & 100 \\
\hline & Adesão & AA & AA & AA & AA & AA & AA & AA & MA & MA & AA \\
\hline \multirow{3}{*}{ CP3 } & $\mathrm{N}$ & 2 & 3 & 3 & 3 & 2 & 2 & 2 & 3 & 3 & 3 \\
\hline & $\%$ & 66,7 & 100 & 100 & 100 & 66,7 & 66,7 & 66,7 & 100 & 100 & 100 \\
\hline & Adesão & MA & AA & AA & AA & MA & MA & MA & AA & AA & AA \\
\hline
\end{tabular}

Fontes. Dados da pesquisa.

O volume de recurso também é expressivo, como pode ser observado na tabela 3 . Em termos reais, as prefeituras garantiram acréscimo de receita no período da ordem de $87,82 \%$, passando de pouco mais de R \$ 20 milhões em 2002 para R \$ 37,81 milhões em 2011. Apesar das variações anuais, é possível perceber nitidamente que houve um comportamento que garantiu (apesar das variações) acréscimos de recursos ao longo do período. Houve acréscimo de recurso acima da inflação da ordem de $87,83 \%$, considerando o primeiro ano (2002) e o último (2011). Portanto, ano a ano essa agenda foi aumentando sua importância dentro do gasto governamental das prefeituras do estado do Mato Grosso.

A variação da receita não teve comportamento linear (Tabela 3). Ao longo do período, é possível notar que na primeira metade da década houve duas quedas expressivas de receita, em $2003(-29,05 \%)$ e 2005 (-9,04\%), quando comparada aos anos anteriores. $\mathrm{Na}$ segunda metade houve apenas uma queda, $-10,71 \%$, no ano de 2009. Essas quedas não comprometeram que no estoque final as prefeituras tivessem feito aporte de recursos importantes nas políticas de esporte e de lazer. 
Tabela 3. Gasto na FDL por parte dos municípios do estado do Mato Grosso de 2002 a 2011 (em milhões).

\begin{tabular}{|l|l|l|l|l|l|l|l|l|l|l|}
\hline FDL & 2002 & 2003 & 2004 & 2005 & 2006 & 2007 & 2008 & 2009 & 2010 & 2011 \\
\hline R $\$$ & 20,1 & 14,3 & 17,9 & 16,3 & 20,9 & 22,9 & 29,2 & 26,1 & 30,7 & 37,8 \\
\hline Var.(\%) & - & $-29,0$ & 25,2 & $-9,0$ & 28,5 & 9,6 & 27,7 & $-10,7$ & 17,5 & 23,3 \\
\hline
\end{tabular}

Fontes. Dados da pesquisa.

É razoável supor que o maior volume de recurso esteja com as cidades da CP1, por conta do maior número $(\mathrm{n}=135)$. Apensar de apresentarem o mesmo número, a $\mathrm{CP} 2$ e $\mathrm{CP} 3$ alternaram sua posição em relação ao total de recurso investido na FDL, como pode ser observado na tabela 4 . Na tabela 3 foram 3 anos de variação negativa no total do gasto na FDL: 2003, 2005 e 2009. Somente na CP1 houve simetria com essa variação. $\mathrm{Na} \mathrm{CP} 2$ a variação negativa foi em 2004, 2006 e 2010. As cidades da CP4 tiveram maior variação negativa ao longo do período: 2003, 2005, 2007, 2009 e 2010.
Dada a autonomia alocativa em matérias em que não há regulação federal para o gasto, como nas políticas públicas de esporte e de lazer, os municípios podem apresentar grande variação alocativa (Souza, 2001). Essa variação pode promover a diversidade ou a desigualdade. Enquanto a diversidade premia a diversidade da política, a desigualdade estabelece padrões desiguais de gasto para municípios com mesma capacidade fiscal. Para essa agenda, não há instrumento normativos para ofertar serviços equitativos (Cavalcante, 2011).

Tabela 4. Gasto na FDL por parte dos municípios do estado do Mato Grosso de 2002 a 2011 por CP (em milhões).

\begin{tabular}{|c|c|c|c|c|c|c|c|c|c|c|c|}
\hline CP & FDL & 2002 & 2003 & 2004 & 2005 & 2006 & 2007 & 2008 & 2009 & 2010 & 2011 \\
\hline CP1 & R $\$$ & 15,4 & 11,4 & 14,4 & 12,8 & 17,0 & 19,1 & 23,9 & 20,8 & 27,8 & 30,1 \\
\cline { 2 - 12 } & Var.(\%) & - & $-25,8$ & 25,6 & $-10,6$ & 32,7 & 12,2 & 25,0 & $-13,1$ & 33,7 & 8,3 \\
\hline \multirow{2}{*}{ CP2 } & R $\$$ & 1,8 & 1,8 & 1,8 & 2,0 & 1,7 & 2,4 & 2,4 & 3,3 & 1,4 & 2,3 \\
\cline { 2 - 12 } & Var.(\%) & - & 0,0 & $-3,4$ & 14,8 & $-17,3$ & 39,8 & 1,8 & 35,9 & $-56,9$ & 62,8 \\
\hline CP3 & R $\$$ & 2,9 & 1,0 & 1,7 & 1,4 & 2,2 & 1,4 & 2,9 & 2,1 & 1,5 & 5,4 \\
\cline { 2 - 11 } & Var.(\%) & - & $-64,9$ & 72,0 & $-20,4$ & 56,7 & $-35,0$ & 107,6 & $-29,6$ & $-28,1$ & 267,7 \\
\hline
\end{tabular}

Fontes. Dados da pesquisa.

A comparação do desempenho dos entes federados na descentralização das políticas de esporte e de lazer, mesma que parcial, entre o governo local e o governo federal pode permitir compreender melhor a capacidade alocativa dos municípios do estado do Mato Grosso. As duas principais políticas top-down na área social do Ministério do Esporte são os programas Segundo Tempo (PST) e Esporte e Lazer da Cidade (PELC). No caso do Ministério do Esporte, a descentralização não é feita de forma prioritária aos municípios, o terceiro setor tem sido um parceiro estratégico do governo federal na implementação dos programas. (Souza Neto, Santos, \& Starepravo, 2015). Como pode ser observado na tabela 5 , o governo federal variou sua capacidade de financiamento dos programas, sendo responsável por $3,44 \%$ ( $\mathrm{R} \$ 44,47$ milhões para $\mathrm{R} \$ 1,48$ milhões) dos recursos no estado em 2011 e 26,39\% (R\$ 21,03 milhões para $\mathrm{R} \$ 5,55$ milhões) em $2002^{12}$.

Apesar da variação, houve perda importante quando consideramos o desempenho do governo federal ao longo do caminho. Portanto, os municípios são muito mais efetivos no gasto do que o governo federal no estado do Mato Grosso. Enquanto os municípios realizaram gasto de 2004 a 2011 na ordem de R \$ 237,26 milhões, o governo federal garantiu ao estado R \$32,36 milhões. Esse resultado é compatível com o que destaca baracho (2000), ao identificar que as prefeituras são muito mais responsivas a determinadas agendas.
12 Para essa análise os dois valores nominais (dos municípios e do governo federal) foram deflacionados pelo mesmo critério. O período deflacionado foi de 2004 a 2011. 
Tabela 5. Evolução das despesas na FDL e das transferências do governo federal de 2004 a 2011.

\begin{tabular}{|c|c|c|c|c|c|c|c|c|c|}
\hline Gasto & Evolução & 2004 & 2005 & 2006 & 2007 & 2008 & 2009 & 2010 & 2011 \\
\hline \multirow{2}{*}{ FDL } & $\mathrm{R} \$$ & 21,03 & 19,13 & 24,57 & 26,92 & 34,38 & 30,70 & 36,06 & 44,47 \\
\cline { 2 - 11 } & Var.(\%) & - & $-9,05$ & 28,48 & 9,57 & 27,68 & $-10,71$ & 17,47 & 23,31 \\
\hline PELC/ & $\mathrm{R} \$$ & 5,55 & 2,22 & 3,9 & 3,07 & 6,42 & 5,37 & 4,35 & 1,48 \\
\cline { 2 - 11 } PST & Var.(\%) & - & $-60,0$ & 75,7 & $-21,3$ & 109,1 & $-16,4$ & $-19,0$ & $-66,0$ \\
\hline
\end{tabular}

Fonte: FDL, dados da pesquisa e PELC, Castro (2016).

No que tange os gastos na FDL, os resultados aqui apresentados vão ao encontro das avaliações de que o fortalecimento da capacidade decisória dos governos subnacionais, especialmente os municípios, é um fator importante à descentralização bottom-up, como destaca Almeida (1995), e garante maior conexão com os interesses locais, como apontam Arretche, Vasquez e Gomes (2012). Porém, no entendimento de Rodden (2005), não é condicional à descentralização obter ganhos de accountability (o maior controle pode exigir maior responsividade em relação às demandas locais). Portanto, não estamos diante de uma regra geral. O resultado apresentado aqui do estoque do gasto das prefeituras nas políticas de esporte e de lazer é particular às relações federalistas que acontecem no estado do Mato Grosso.

Não só a inação foi descartada como um comportamento esperado, diante dos constrangimentos normativos no âmbito da federação, como a proatividade das prefeituras nesse campo ficou destacada pelo número de adesões na FDL e pelos valores envolvidos na operação. Resultado esse que vai ao encontro de outros achados sobre o tema da descentralização bottom up das políticas públicas de esporte e de lazer (Santos, Starepravo, \& Canan, 2018; Santos, 2018a; Santos, 2018b; Santos; Starepravo, 2018). A preferência alocativa (tomada de decisão sobre onde investir) aponta para participação dos municípios do estado do Mato Grosso no financiamento das políticas de esporte e de lazer.

\section{Conclusão}

Conhecer o gasto descentralizado nas políticas públicas de esporte e de lazer em um dado período é fundamental para a implementação de uma agenda ativa e decisões capazes de alcançar as demandas

\section{Referências}

Afonso, J. R. R., Lobo, T. (1996). Descentralização fiscal e participação em sociais. Ao fazer isso em relação as prefeituras do Estado de mato Grosso, o resultado aponta para um fenômeno importante às políticas públicas de esporte e de lazer no Brasil. No estado do Mato Grosso há uma forte inclinação das prefeituras em descentralizar bottom up o gasto na FDL. A média de adesão na segunda metade do período foi de $91,77 \%$.

Em políticas de livre alocação de recursos ocorre diversidade e ou desigualdade na oferta da política. A diversidade está associada ao custo da política a ser implementada. Determinadas opções podem ser mais baratas do que outras e não significam oferta desigual. No entanto, as opções podem ser produzidas a partir de constrangimentos fiscais, o que pode levar a oferta desigual. A descentralização bottom up não significa redução da desigualdade, podendo, inclusive, aumentá-la. É preciso destacar que mesmo nas cidades pequenas (abaixo de $50 \mathrm{mil} / \mathrm{hab}$.) houve elevada adesão.

Os dados aqui trabalhados não permitem fazer conjecturas sobre o impacto da descentralização. Mas é uma questão importante a ser aprofundada por outras pesquisas, pois têm impacto na oferta da política na federação. A correção dessas desigualdades (Inciso 3 do Art. 3 da CF/88) deve ser realizada pelo governo federal, daí sua importância. Sendo assim, os resultados aqui descritos abrem espaços para novas investigações no sentido de melhor compreender a alocação dos recursos na FDL

\section{Agradecimento}

Agência de Fomento: Rede Cedes, Ministério da Cidadania.

\section{experiências democráticas retardatárias. Planejamento e Políticas Públicas, 14, 3-36.}

Almeida, B. S., Marchi Júnior, W. (2010). O financiamento dos programas federais de esporte e lazer no Brasil (2004 a 2008). Movimento, 16(4), 73-92. 
Almeida, M. H. T. (1995). Federalismo e políticas sociais. Revista Brasileira de Ciências Sociais, 10(28), 88-108.

Arretche, M., Vasquez, D., Gomes, S. (2012) Descentralização e autonomia: deslocando os termos do debate. In: A. G. Lavalle (Org.). O Horizonte da política: questões emergentes e agendas de pesquisa (pp. 29-62). São Paulo: UNESP.

Athayde, P. F. A. O ornitorrinco de chuteiras: determinantes econômicos da política de esporte $e$ lazer do Governo Lula e suas implicações sociais. (2014). Tese, Universidade de Brasília.

Athayde, P. F. A., Mascarenhas, F., Salvador, E. (2015). Primeiras aproximações de uma análise do financiamento da política nacional de esporte e lazer no Governo Lula. Revista Brasileira de Ciências do Esporte, 37(1), 2-10.

Baracho, M. A. P. (2000). A importância da gestão de contas públicas municipais sob a premissa da governance e accountability. Revista do Tribunal de Contas do Estado de Minas Gerais, 34(1), 129-161.

Castro, S. B. E. Políticas públicas para o esporte e lazer e o ciclo orçamentário brasileiro (2004-2011): prioridades e distribuição de recursos durante os processos de elaboração e execução orçamentária. (2016). Tese, Universidade Federal do Paraná.

Castro, S. B. E., Scarpin, J .E., Souza, D. L. (2017). Financiamento público e esporte educacional: uma análise do processo orçamentário do programa "Segundo Tempo" (2004-2011). Motrivivência, 29(52), 136-156.

Corrêa, A. J., Moraes e Silva, M., Mezzadri, F., Cavichiolli, F. R. (2014). Financiamento do esporte olímpico de verão brasileiro: mapeamento inicial do programa Bolsa Atleta (2005-2011). Pensar a Prática, 17(4), 1-15.

Diniz, R. S., Silva, L. P. (2016). O ICMS esportivo e o financiamento das políticas municipais de esporte em minas gerais. Movimento, 22(4), 1223-1236.

Furtado, S., Effting, E. R. M., Castro, S. B. E., Souza, D. L. (2016). O Financiamento do esporte em Santa Catarina: o caso do Fundesporte nos anos de 2011 e 2012. Motrivivência, 28(47), 145-159.

Grasso, R. M. F. P., Isayama, H. F. (2011). Financiamento e políticas públicas de esporte e lazer: uma análise da gestão no município de Santarém/PA
(2005-2012). Revista Brasileira de Ciência e Movimento, 25(1), 151-167.

Green, M. (2007). Policy transfer, lesson drawing and perspectives on elite sport development systems. International Journal of Sport Management and Marketing, 2(4), 426-441.

Hall, P., Taylor, R. (2003). As três versões do neoinstitucioalismo. Lua Nova, 58, 193-222.

Hoekman, R., Van Der Roest, J., Van Der Poel, H. (2018). From welfare state to participation society? Austerity measures and local sport policy in the Netherlands. International Journal of Sport Policy and Politics, 10(1), 131-146.

Melo, M. A. (1998). Crise federativa, guerra fiscal e hobbestanismo municipal: efeito perversor da descentralização? São Paulo em Perspectiva, 10(3), $10-20$.

Moraes, D. P. (2006). Arrecadação tributária municipal: esforço fiscal, transferências e Lei de Responsabilidade Fiscal. Dissertação, Fundação Getúlio Vargas.

Pavlík, M. Economic Characteristic of Non-Profit Sport Clubs and Their Relations with Municipalities and Sport Federations (June 4, 2013). Disponível em: <https://ssrn.com/abstract=2274455> Acesso em: 5 de abr. 2018.

Pavlík, M., Vries, M. S. (2014). The Voucher System as an Alternative for Allocating Sports Grants. Central European Journal of Public Policy, 8(2), 4-27.

Ribeiro, O. C. F. (2012). Um estudo das políticas públicas de lazer de Brotas/SP. Tese, Universidade Estadual de Campinas.

Rocha, C. V. (2011). Federalismo. Civitas, 11(2), 323-338.

Rodden, J. (2005). Federalismo e descentralização em perspectiva comparada: sobre significados e medidas. Revista Sociologia e Política, 24, 9-27.

Ronglan, L. T. (2015). Elite sport in Scandinavian welfare states: legitimacy under pressure? International journal of sport policy and politics, 7(3), 345-363.

Sabatier, P. (1986). Top-down and bottom-up approaches to implementation research: a critical 
analysis and suggested synthesis. Journal of Public Policy, 6(1), 21-48.

Santos, A. M. P. (2011). Federalismo no Brasil: uma abordagem da perspectiva dos Municípios. Revista de Direito da Cidade, 3(1), 95-123.

Santos, E. S.; Santos, A. G. (2018). Participação dos municípios do estado do Espírito Santo nos gastos da Função Desporto e Lazer. Conexões: Educ. Fís., Esporte e Saúde, 16(3), 312-324.

Santos, E. S. (2017). Análise do Desempenho do Edital de 2012 do PELC. Licere, 20, 41-58.

Santos, E. S. (2018). Gasto na Função Desporto e Lazer pelos municípios do estado do Maranhão. Revista Intercontinental de Gestão Desportiva, 8, 138-150.

Santos, E. S. (2016). Governo do estado e descentralização Programa Segundo Tempo. Pensar a Prática, 19, 398-406.

Santos, E. S., Canan, F., Starepravo, F. A. (2018). Investments in the sport and leisure function by municipalities in Bahia from 2002 to 2011. Journal of physical education, 29, 1-10.

Santos, E. S., Hirata, E. (2017). Investimento na função desporto e lazer por níveis de Índice de Desenvolvimento Humano. Caderno de Educação Física, 15, 49-55.

Santos, E. S., Starepravo, F. A. (2018). PoliticalAdministrative Structure of Municipal Governments in Piauí and Investment in Sport and Leisure. Licere, 21, 166-183.

Santos, E. S., Starepravo, F. A., Hirata, E. (2018). Perfil das Prefeituras que descentralizaram o PELC de 2003 a 2012. Licere, 21, 71-88.

Silva, T. D., Couto, A. M. S., Santos, M. L. A. (2014). Analysis of the administrative structure and financial sport in IPIAU-BA county. Podium: Sport, Leisure And Tourism Review, 3(3), 77-88.

Silva, T. D., Santana, T. A., Silva, R. C. (2015). O planejamento e a estrutura financeira das políticas públicas de lazer no estado da Bahia. Coleção Pesquisa Em Educação Física, 14(4), 121-130.

Souza Neto, M., Santos, E. S., Starepravo, F. A. (2015). Programa Segundo Tempo e o vazio assistencial na região Nordeste. Movimento, 21(3), 759-771.

Souza, C. (2003). Estado do campo da pesquisa em políticas públicas no Brasil. Revista Brasileira de Ciências Sociais, 18(51), 15-20.

Sperotto, F. Gasto social nos anos 1990: o caso dos municípios gaúchos com mais de 100 mil habitantes (2009). Planejamento e Políticas Públicas, Brasília, 23, 71-118.

Starepravo, F. A. (2011). Políticas públicas de esporte e lazer no Brasil: aproximações, intersecções, rupturas e distanciamentos entre os subcampos político/burocrático e científico/acadêmico. Tese, Universidade Federal do Paraná.

Taks, M., Késenne, S. (2000). Tie Economic Significance of Sport in Flanders. Journal of Sport Management, 14, (4), 342-365.

Venturim, L. F., Borges, C. N. F., Silva, D. S. (2013). Estratégias de gestão pública na prefeitura de Vitória/ES? O Pelc e a intersetorialidade das ações. Licere, 16(4), 1-38.

Veronez, L. F. C. Quando o Estado Joga a Favor do Privado: As Políticas de Esporte após a Constituição Federal de 1988. (2005). Tese, Universidade Estadual de Campinas.

Widdop, P., King, N., Parnell, D., Cutts, D., Millward, P. (2018). Austerity, policy and sport participation in England. International Journal of Sport Policy and Politics, 10(1), 7-24.

Yuval, F. (2011). Information theory and municipal policy in sport: empirical evidence from an Israeli case study. Sport in Society, 14(1), 53-68. 